\title{
Single-mode coherent synchrotron radiation instability of a bunched beam
}

\author{
S. Heifets \\ Stanford Linear Accelerator Center, Stanford University, Stanford, California 94309, USA
}

(Received 28 March 2003; published 13 August 2003)

\begin{abstract}
The coherent synchrotron radiation instability may be driven at the shielding threshold by a single mode excited in the beam pipe. Such a problem may have general interest for other one-mode dominated problems arising in plasma, free-electron laser theory, and microwave instability. The problem may have practical interest if the steady state exists but requires the study of the nonlinear regime of instability. The results of the study, both analytic and numerical, are presented in the coasting beam approximation and for a bunched beam.
\end{abstract}

DOI: 10.1103/PhysRevSTAB.6.080701

PACS numbers: 29.27.Bd

\section{INTRODUCTION}

Coherent synchrotron radiation (CSR) can cause beam instability. In the previous paper [1] we studied the CSR instability [2] of a coasting beam interacting with a single waveguide mode which may define beam dynamics close to the shielding threshold. The argument for using the coasting beam model, besides the substantial simplification of the theory, was based on the fact that the wavelength of the relevant perturbation is small as compared to the bunch length. The instability was studied both in linear and nonlinear regimes. It was shown that even over a large time scale (comparable or larger than the damping time) there is no saturation of the instability: the amplitude of the perturbation keeps growing slowly but steadily.

On the large scale time intervals the approach has to be reconsidered by taking the synchrotron motion into account, thus providing the impetus for this study. Another motive is provided by the recent proposal to build a dedicated storage ring for the generation of infrared coherent radiation [3]. For existing machines, the linear regime is limited to the time intervals of less than a microsecond. Therefore, it is important to consider the nonlinear regime of the instability.

This paper is organized in the following way. First, the basic equations describing beam dynamics and the interaction of the beam with the excited electromagnetic (EM) wave are derived. Then, it is shown that although a steady-state solution exists, it is unstable and the growth rate for a bunched beam is determined in the linear approximation. The next two sections present the qualitative analysis of the nonlinear regime and estimate the growth rate. Then, the results of numeric simulations are presented and compared with the qualitative analysis.

\section{BASIC EQUATIONS}

Beam dynamics is described neglecting the nonlinearity of the rf field and assuming that the nonlinearity is dominated by the beam-wave interaction $V_{B}(z, t)$,

$$
\frac{d z}{d t}=-\eta c \delta, \quad \frac{d \delta}{d t}=\frac{\omega_{s}^{2}}{\eta c} z+V_{B}(z, t),
$$

where $z$ is the position of a particle along the bunch, $\delta$ is the relative energy offset, $\omega_{s} /(2 \pi)$ is the synchrotron frequency, and $\eta$ is the momentum compaction factor. The EM wave excited by the beam in the waveguide is defined by the wave frequency $\omega_{w}$, the wave vector $q_{w}=$ $\omega_{w} / c$, the loss factor per unit length $\chi$, and the group velocity $\beta_{g} . V_{B}=w_{B}+$ c.c. is defined [1] by the distribution function $f, \int d z d \delta f(z, \delta, t)=1$ (see Appendix A)

$$
\begin{aligned}
w_{B}(z, t)= & -\frac{r_{e} N_{B} c^{2}}{\gamma}\left(1-\beta_{g}\right) \chi \int_{-\infty}^{t} d t^{\prime} d \delta^{\prime} f \\
& \times\left[z+c\left(t-t^{\prime}\right)\left(1-\beta_{g}\right), \delta^{\prime}, t^{\prime}\right] e^{-i\left(1-\beta_{g}\right) \omega_{w}\left(t-t^{\prime}\right)} .
\end{aligned}
$$

Here $N_{B}$ is the number of particles per bunch, and $\gamma$ is the relativistic parameter of the beam (not to be confused with the radiation damping $\gamma_{S R}=1 / \tau_{S R}$ below).

It is convenient to use dimensionless variables $(\tau, \zeta, P)$ and interaction $V(\zeta, \tau)$ introducing $\tau=\mu t$,

$$
\zeta=q_{w} z, \quad P=-\frac{\eta \omega_{w}}{\mu} \delta, \quad V\left(\zeta, \tau_{B}\right)=\frac{\eta \omega_{W}}{\mu^{2}} V_{B}(z, t),
$$

where $\mu$ depends on the peak density in a bunch $n_{B}=$ $N_{B} /\left(\sigma_{B} \sqrt{2 \pi}\right)$.

$$
\left(\frac{\mu}{c}\right)^{3}=\frac{r_{e} n_{B}}{c \gamma}\left(1-\beta_{g}\right) \chi \eta \omega_{w} .
$$

It is convenient to use notations

$\kappa=\left(\frac{\omega_{w}}{\mu}\right)\left(1-\beta_{g}\right), \quad \Delta=\frac{\eta \omega_{w} \delta_{0}}{\mu}, \quad \Gamma=\frac{\gamma_{S R}}{\mu}, \quad \Omega=\frac{\omega_{s}}{\mu}$,

where $\delta_{0}$ is the rms of the relative energy spread. The corresponding Hamiltonian $H$ is

$$
H(\zeta, P, \tau)=\frac{P^{2}}{2}+\frac{\Omega^{2} \zeta^{2}}{2}+U(\zeta, \tau) .
$$


The potential $U$ is defined by $d U / d \zeta=V, V(\zeta, \tau)=$ $w(\zeta, \tau)+$ c.c., where

$$
w(\zeta, \tau)=-\lambda^{3} \int_{-\infty}^{\tau} d \tau^{\prime} d P^{\prime} F\left[\zeta+\left(\tau-\tau^{\prime}\right) \kappa, P^{\prime}, \tau^{\prime}\right] e^{-i \kappa\left(\tau-\tau^{\prime}\right)} .
$$

Here

$$
\lambda^{3}=\sqrt{2 \pi} \frac{\Delta}{\Omega},
$$

and the integration over $\tau^{\prime}$ from $-\infty$ implies that a small $\epsilon \rightarrow+0$ is added to the exponent.

The distribution function $F(\zeta, P, \tau)$ is normalized, $\int d P d \zeta F(\zeta, P, \tau)=1$ and satisfies the Fokker-Planck equation

$$
\frac{\partial F}{\partial \tau}+P \frac{\partial F}{\partial \zeta}-\left[\Omega^{2} \zeta+V(\zeta, \tau)\right] \frac{\partial F}{\partial P}=\Gamma \frac{\partial}{\partial P}\left[\Delta^{2} \frac{\partial F}{\partial P}+P F\right] .
$$

The Fourier harmonics $\tilde{w}(q, \tau)$

$$
w(\zeta, \tau)=\int \frac{d q}{2 \pi} \tilde{w}(q, \tau) e^{i q \zeta}
$$

is related to $F$,

$$
\frac{\partial \tilde{w}(q, \tau)}{\partial \tau}=i(q-1) \kappa \tilde{w}(q, \tau)-\lambda^{3} \int d \zeta d P e^{-i q \zeta} F(\zeta, P, \tau) .
$$

Note that $\Delta / \Omega=q_{w} \sigma_{B} \gg 1$ for the high frequency waves with the wavelength $2 \pi / q_{w}$ which is small in comparison to the rms bunch length $\sigma_{B}$.

Parameters of four typical machines are given in Table I.

\section{STEADY-STATE SOLUTION}

The Fokker-Planck equation (9) has a formal steadystate solution which, after integration over $P$, takes the form

$$
F(\zeta)=\frac{1}{|N|} e^{-\left(1 / \Delta^{2}\right)\left[\left(\Omega^{2} \zeta^{2} / 2\right)+U(\zeta)\right]},
$$

where $|N|$ is a normalization constant and $U(\infty)=0$.

This can be solved by iterations starting with a Gaussian bunch. Calculations show that iterations for

TABLE I. Parameters for several machines.

\begin{tabular}{ccccc}
\hline \hline & LER & HER & ALS & VUV NSLS \\
\hline$\mu, 1 / \mu \mathrm{s}$ & 7.4 & 2.4 & 17.9 & 22.7 \\
$\kappa$ & 143.0 & 125.0 & 124.0 & 138.0 \\
$\Omega$ & $4.2 E-3$ & $15 . E-3$ & $2.4 E-3$ & $3.3 E-3$ \\
$\Delta$ & 0.067 & 0.85 & 0.02 & 0.012 \\
\hline \hline
\end{tabular}

typical parameters converge very well. However, the linear analysis given below shows that such a steady-state solution is unstable.

Still, the question remains whether there is a timeindependent solution $F(\zeta, \tau)=F(\zeta)$ with a large amplitude of perturbation $V(\zeta)$.

Equation (7) can be written as

$$
\begin{aligned}
& V(\zeta)=A(\zeta) e^{i \zeta}+\text { c.c., } \\
& A(\zeta)=-\frac{\lambda^{3}}{\kappa} \int_{\zeta}^{\infty} d \zeta^{\prime} e^{-i \zeta^{\prime}} F\left(\zeta^{\prime}\right) .
\end{aligned}
$$

Hence, the steady-state solution is given by the system of equations

$$
\begin{gathered}
\frac{d U(\zeta)}{d \zeta}=A(\zeta) e^{i \zeta}+\text { c.c., } \quad \frac{d A(\zeta)}{d \zeta}=\frac{\lambda^{3}}{\kappa} e^{-i \zeta} F(\zeta), \\
F(\zeta)=\frac{1}{|N|} e^{-\left(1 / \Delta^{2}\right)\left[\left(\Omega^{2} \zeta^{2} / 2\right)+U(\zeta)\right]},
\end{gathered}
$$

$$
|N|=\int d \zeta e^{-\left(1 / \Delta^{2}\right)\left[\left(\Omega^{2} \zeta^{2} / 2\right)+U(\zeta)\right]} .
$$

The system is suitable for simulations and gives $U(\zeta)$ oscillating with a constant amplitude for all $\zeta$. The same solution can be found analytically. However, a solution of the form

$$
U(\zeta)=a \cos \left(\zeta-\zeta_{0}\right)
$$

where $a=$ const violates casuality and is not acceptable. This argument shows, but does not prove, that a steadystate solution, which is nonzero only behind the bunch, does not exist.

\section{LINEARIZED VLASOV EQUATION WITH THE SYNCHROTRON MOTION}

In this section, we show that the steady-state solution with the zero amplitude perturbation is unstable: a small perturbation grows in time exponentially.

With synchrotron motion taken into account, there is no periodicity over $\zeta$ even for a bunch interacting with a single EM wave. The Fourier harmonics of the distribution function are

$$
F(\zeta, P, \tau)=\int \frac{d q}{2 \pi} e^{i q \zeta} \tilde{F}(q, P, \tau) .
$$

Assuming the time dependence of the harmonics in the form $\tilde{w}(q, \tau)=\hat{w}(q) e^{-i \nu \tau}, \tilde{F}(q, P, \tau)=\hat{F}(q, P) e^{-i \nu \tau}$ with amplitudes $\hat{F}(q, P)$ and $\hat{w}(q)$, respectively, we get from Eq. (11)

$$
\hat{w}(q)=-i \lambda^{3} \frac{\int d P \hat{F}[q, P]}{(q-1) \kappa+\nu} .
$$


In zero approximation, the distribution function is $F_{0}(\zeta, P)$

$$
F_{0}(\zeta, P)=\frac{1}{Z} e^{-\left(1 / \Delta^{2}\right)\left[\left(P^{2} / 2\right)+\left(\Omega^{2} \zeta^{2} / 2\right)\right]},
$$

where the normalization constant $Z=2 \pi \Delta^{2} / \Omega$. The contribution of $F_{0}$ to Eq. (18) is exponentially small when $q \simeq 1$.

In linear approximation, $\quad F(\zeta, P, \tau)=F_{0}+$ $\left[f(\zeta, P) e^{-i \nu \tau}+\right.$ c.c. $]$, and $\hat{F}(q, P)$ in Eq. (18) can be replaced by $\hat{f}(q, P)$.

Equation (9) is reduced to the Vlasov equation for $f(\zeta, P, \tau)$ :

$$
\frac{\partial f}{\partial \tau}+P \frac{\partial f}{\partial \zeta}-\Omega^{2} \zeta \frac{\partial f}{\partial P}=V(\zeta, \tau) \frac{\partial F_{0}}{\partial P} .
$$

Equation (20) can be easily solved using the method of characteristics,

$$
\begin{gathered}
f(\zeta, P, \tau)=-\frac{F_{0}(\zeta, P)}{\Delta^{2}} \int_{-\infty}^{\tau} d \tau^{\prime}[\Omega \zeta \sin \phi+P \cos \phi], \\
V\left[\zeta \cos \phi-\frac{P}{\Omega} \sin \phi, \tau^{\prime}\right],
\end{gathered}
$$

where $\phi=\Omega\left(\tau-\tau^{\prime}\right)$.

Substitute Eqs. (19) and (21) in the definition of the Fourier harmonics

$$
\hat{f}(q, P)=\int d \zeta e^{-i q \zeta+i \nu \tau} f(\zeta, P, \tau) .
$$

Integration over $P$ and $\zeta$ gives

$$
\int d P \hat{f}(q, P)=\frac{i q}{\Omega} \int \frac{d q^{\prime}}{2 \pi} \hat{V}\left(q^{\prime}\right) e^{-(1 / 2)(\Delta / \Omega)^{2}\left(q-q^{\prime}\right)^{2}} S\left(q, q^{\prime}\right),
$$

where

$$
S\left(q, q^{\prime}\right)=\int_{0}^{\infty} d \tau e^{i \nu \tau} \sin (\Omega \tau) e^{-(\Delta / \Omega)^{2} q q^{\prime}[1-\cos (\Omega \tau)]} .
$$

Combining Eqs. (18) and (23), we get

$$
\hat{V}(q)=\frac{\lambda^{3} q}{\Omega[\nu+(q-1) \kappa]} \int \frac{d q^{\prime}}{2 \pi} \hat{V}\left(q^{\prime}\right) e^{-(1 / 2)(\Delta / \Omega)^{2}\left(q-q^{\prime}\right)^{2}} S\left(q, q^{\prime}\right) .
$$

As was mentioned above, the ratio $\Omega / \Delta=$ $1 /\left(q_{w} \sigma_{b}\right) \ll 1$ for short wavelength perturbations. Therefore, the integral in Eq. (25) can be estimated taking factors $\hat{V}\left(q^{\prime}\right) S\left(q, q^{\prime}\right)$ at $q^{\prime}=q$. Equation (25) then gives the dispersion equation

$$
1=\frac{\lambda^{3} q}{\Delta \sqrt{2 \pi}} \frac{S(q, q)}{\nu+(q-1) \kappa} .
$$

The function $\Omega S(q, q)$ is a function of two parameters,

$$
S(q, q)=\frac{1}{\Omega} \int_{0}^{\infty} d x \sin (x) e^{i(\nu / \Omega) x} e^{-b^{2}(1-\cos x)},
$$

where $b=q \Delta / \Omega \gg 1$. The main contribution to the integral Eq. (27) is given by $|x| \ll 1$. The other contributions come from the narrow vicinities of $x_{k}=2 \pi k$, $k=$ integer, which cancel each other provided there are no resonances $\nu / \Omega=$ integer. Therefore, for $\Omega / \Delta \ll 1$, the integral can be simplified,

$$
S(q, q)=\frac{\Omega}{(q \Delta)^{2}} G\left[\frac{\nu}{q \Delta}\right]
$$

where

$$
G[a]=\int_{0}^{\infty} x d x e^{i a x} e^{-x^{2} / 2} .
$$

For large $|a| \gg 1, G(a) \simeq-\left(1 / a^{2}\right)$, and Eq. (26) gives

$$
\nu^{2}[\nu+(q-1) \kappa]=-\frac{\lambda^{3} q}{\sqrt{2 \pi}} \frac{\Omega}{\Delta} .
$$

Using definition Eq. (8), we get for $q=1$,

$$
\nu^{3}=-\frac{\lambda^{3} \Omega}{\Delta \sqrt{2 \pi}}=-1
$$

The growth rate $\operatorname{Im}(\nu)=\sin [\pi / 3]$ exactly corresponds to the growth rate defined before [1] for a cold beam (i.e., for $\Delta \ll \nu)$. The synchrotron motion does not change the result provided $\Omega / \Delta \ll 1$, i.e., for the short wavelengths.

The value $q=1$ corresponds to the modulation $V(\zeta, \tau) \propto e^{i(\zeta-\nu \tau)}$ and the perturbation of the distribution function of the form $F(\zeta, P, \tau) \propto e^{i \zeta}=e^{i q_{w} z}$, i.e., the spatial modulation with the wavelength of the EM wave.

For $q \neq 1$, the growth rate is reduced provided $\kappa \gg 1$. The function $\operatorname{Im}[\nu]$ is shown in Fig. 1 as a function of $q$ for $\kappa=25$.

Equation (30) gives for $1 \gg|q-1| \kappa \gg|\nu| / \kappa$,

$$
\nu^{2}=-\frac{1}{(q-1) \kappa} \text {. }
$$

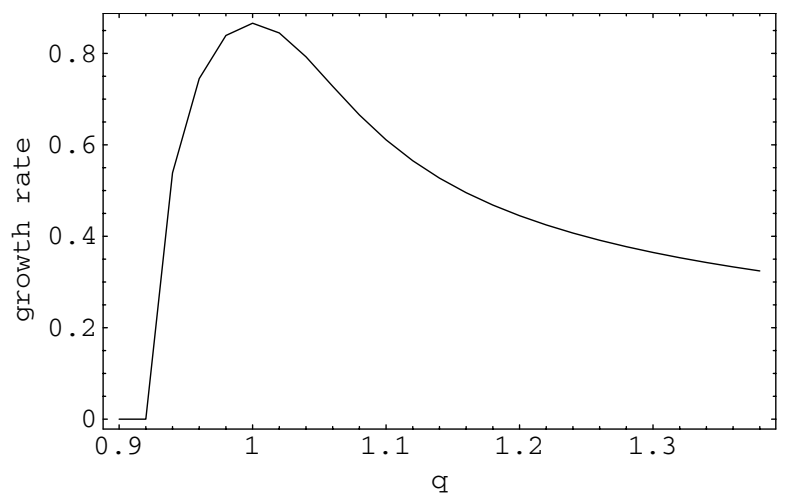

FIG. 1. The growth rate $\operatorname{Im}[\nu]$ as a function of the detuning for $\kappa=25$. 
Equation (32) gives the estimate for the width of the resonance and shows that instability is substantial only for harmonics of the distribution function $F(q)$ in the narrow vicinity of $q=1$. From these results, it is clear that, in the linear approximation, different EM modes in the beam pipe can be considered independently.

\section{PARTICLE MOTION IN THE NONLINEAR REGIME: QUALITATIVE ANALYSIS}

Fast initial growth predicted by the linear theory is limited to a short period of time of the order of the inverse growth rate, $\tau \simeq 1, t \simeq 1 / \mu$. After that, growth of the amplitude is substantially nonlinear. For small time intervals, small as compared with the period of synchrotron oscillations, the nonlinear beam dynamics is the same as for the case of a coasting beam [1]. Describing nonlinear behavior at longer times we found that it is necessary to distinguish between short-range and long-range nonlinear regimes. The first regime can be considered similarly to the analysis given for the coasting beam [1] and is presented in this section. In spite of this similarity, the growth rate in this regime is quite different from the one for the coasting beam and depends on $\Omega$. Analysis and simulations of the second regime are given in the following sections.

As shown above, in the linear regime the beam-wave interaction with a single mode can be written as $V(\zeta, \tau)=$ $a q_{0} \sin \left(q_{0} \zeta-\nu \tau\right)$ with the wave vector $q_{0} \simeq 1$ and the amplitude $a$ exponentially growing in time. We assume that in the nonlinear regime $V(\zeta, \tau)$ has the same form where the mode frequency $\nu(\tau)$ and the amplitude $a(\tau)$ are real, but the amplitude $a$ and the tune $\nu$ may vary slowly in time.

Interaction with such a mode can be described by the system of equations equivalent to the Fokker-Planck equation (9),

$$
\dot{\zeta}(\tau)=P, \quad \dot{P}(\tau)+\Omega^{2} \zeta=-\Gamma P-a q_{0} \sin \left(q_{0} \zeta-\nu \tau\right) .
$$

The quantum fluctuations can be taken into account by adding a random force to the right-hand side of the second equation but can be neglected for time intervals smaller than the damping time.

Equation (33) corresponds to the motion of a particle in the potential

$$
U(\zeta, \tau)=\frac{\Omega^{2} \zeta^{2}}{2}+a\left[1-\cos \left(q_{0} \zeta-\nu \tau\right)\right]
$$

The potential has minima at the points defined by the equation

$$
\zeta+\frac{q_{0} a}{\Omega^{2}} \sin \left(q_{0} \zeta-\nu \tau\right)=0
$$

The potential is a chain of micropotential wells. For $a \gg$ $\Omega^{2}$, they are centered at

$$
\zeta_{k}(\tau) \simeq \frac{1}{q_{0}}\left(1-\frac{\Omega^{2}}{\omega^{2}}\right)(\nu \tau+2 \pi k), \quad k=\text { integer, }
$$

where $\omega^{2}=\Omega^{2}+q_{0}^{2} a$. Equation (36) is valid for $\left.(\Omega / \omega)^{2} \mid \nu \tau+2 \pi k\right) \mid<1$.

For a fixed $\tau$ and large $a \gg 1$, there are approximately

$$
n_{\mathrm{well}} \simeq \frac{a q_{0}^{2}}{\pi \Omega^{2}}
$$

potential wells within the distances $|\zeta|<a$ separated by $\Delta \zeta=2 \pi / q_{0}$; see Fig. 2 .

In a linear approximation, the wave amplitude $a(\tau)$ grows to $a \simeq 1$ much faster than the synchrotron period. Hence, initially, position and momentum of a particle do not change. Particles remain distributed approximately uniformly in the phase space within $|\zeta|<\Delta / \Omega$ and $|P|<\sqrt{2 \Delta}$.

Then, trapped particles start synchrotron oscillations with the amplitude $z_{\max }<\pi$. The frequency of small oscillations is

$$
\omega=\sqrt{a+\Omega^{2}},
$$

and the maximum momentum of a trapped particle is of the order of $P_{\max }=z_{\max } \omega$. Note that the frequency of oscillations of a trapped particle $\omega$ may be large compared to the synchrotron frequency $\Omega$ for amplitudes $a \gg 1$.

The depth of the potential wells is proportional to the amplitude $a$. The energy of a particle $H=\omega J$, where action $J=$ const if $a(\tau)$ changes adiabatically. Because $H \simeq \omega^{2} z^{2} \simeq P^{2}$, the amplitude of particle oscillations $z$ and the energy spread of trapped particles $\Delta_{\mathrm{rms}}(\tau)=$ $\sqrt{\left\langle P^{2}\right\rangle-\langle P\rangle^{2}}$ scale with the amplitude $|a(\tau)|$ as

$$
z \propto \sqrt{\frac{1}{\omega}} \propto a^{-1 / 4}, \quad \Delta_{\mathrm{rms}}(\tau) \propto \sqrt{\omega} \propto a^{1 / 4} .
$$

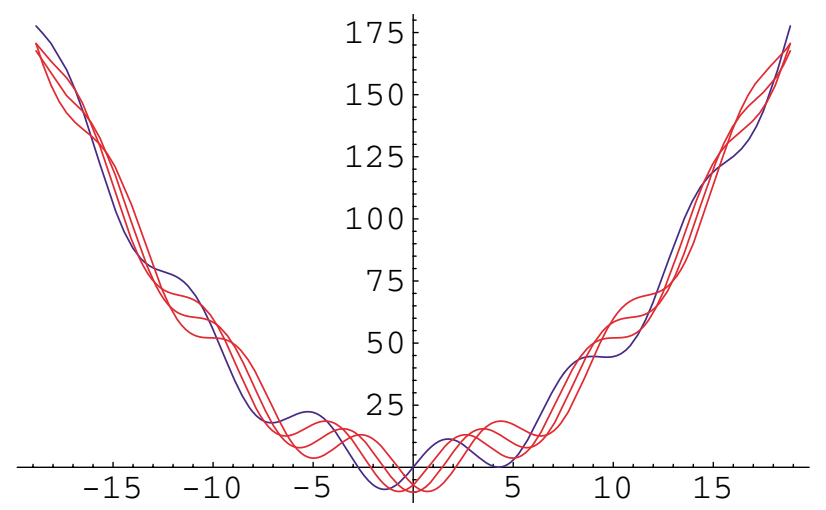

FIG. 2. (Color) The total potential $U_{\text {tot }}=\zeta^{2} / 2+a \sin (\zeta-\nu \tau)$ for $\nu \tau=(0, \pi / 4, \pi / 2,3 \pi / 4)$ and $a=10$. A particle trapped in the local minimum remains trapped and moves with the minimum until it reaches large $\zeta \simeq a$. 

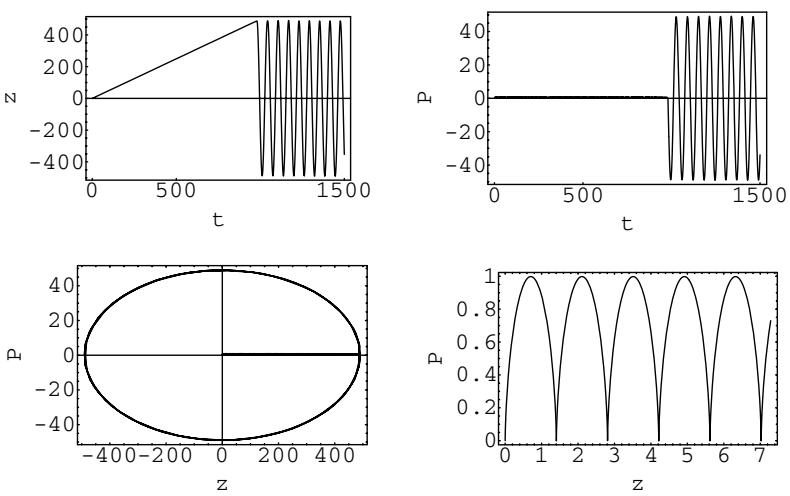

FIG. 3. Trajectory of a particle defined by Eq. (33) with a constant amplitude $a=5.0$. The phase plane is zoomed at small $0<\tau<15$ (bottom right panel) indicating oscillations of trapped and drifting particles. Parameters are shown in the figure. The drift time and the amplitude are in agreement with the estimates $z_{\mathrm{dr}} \simeq a / \Omega^{2},|P| \simeq \Omega z_{\mathrm{dr}}$, and $\tau_{\mathrm{dr}} \simeq z_{\mathrm{dr}} / \nu$. At small $\tau, \omega \simeq \sqrt{a}$ and the shift in $\zeta$ per period is $2 \pi \nu / \omega$.

In addition to the oscillations, a particle trapped in the micropotential well drifts with the potential well at the rate $d \zeta / d \tau=\nu$ as is clear from Fig. 2. It is easy to see that a particle can be trapped as long as $|\zeta|<z_{\mathrm{dr}}=a / \Omega^{2}$.

The condition of adiabaticity implied here requires that the shift of a well minimum $\Delta \zeta \simeq \nu \Delta T$ during one period of oscillations of trapped particles $\Delta T=2 \pi / \omega$ is small compared to the well separation $2 \pi$. That means $\omega \gg \nu$ and is justified for large $a \gg \nu^{2}$.

The time of the drift to the amplitude $z_{\mathrm{dr}}$ is $\tau_{\mathrm{dr}}=$ $a /\left(\nu \Omega^{2}\right)$ and the energy of a trapped particle increases in the rf potential well to large values $U_{\mathrm{dr}}=(a / \Omega)^{2}$.

The depth of a microwell $\Delta U_{k}(\tau)=U\left(\zeta_{k} \mp \pi\right)-$ $U\left(\zeta_{k}\right)$, in which particles were initially trapped at $\zeta_{k}(0)$, decreases when $\zeta_{k}(\tau)>\left|\zeta_{k}(0)\right|$ provided $a(\tau)$ grows slower than $z_{k}(\tau)$ increases. In this case, the microwell population would be reduced with time by releasing particles. The released particles start synchrotron oscillations with large amplitude $z_{\mathrm{dr}}$ and momentum $|P| \simeq \Omega z_{\mathrm{dr}}$.

This qualitative picture is confirmed by numeric calculations of a trajectory defined by the equations of motion (33) with fixed $a=5.0$ and zero initial conditions. The result and other parameters are shown in Fig. 3.

Because the frequency of the synchrotron oscillations and the tune $\nu$ are not commensurate, the released particles may be trapped again mostly due to the radiation damping. If damping is strong, particles are trapped and released in a pattern similar to relaxation oscillations.

\section{Growth rate in the nonlinear regime for $\tau<\tau_{\mathrm{dr}}$}

The distances between macrobunches released from the microwells depend on time. Such bunches are not equidistant as in the case of trapped particles. Additionally, such particles have large energy spread and their interaction with the wave is reduced by Landau damping.
Therefore, interaction of such particles with the wave is small. The wave grows mostly due to its interaction with trapped particles, which can be approximately described in the following way.

At large amplitudes $a \gg \Delta^{2}$, particles trapped in the microwells can be considered as macroparticles located at the minima $\zeta_{k}$; see Eq. (36).

Therefore, we can write an approximate expression for the distribution function $F(\zeta, \tau)=\int d P F(\zeta, P, \tau)$ as

$$
F(\zeta, \tau)=\sum_{k} N_{k}(\tau) \delta\left[\zeta-\zeta_{k}(\tau)\right]
$$

The initial microbunch population is defined by $F(\zeta, 0) \propto$ $\exp \left[-(1 / 2)\left(\Omega \zeta_{k} / \Delta\right)^{2}\right]$. There is substantial population

$$
N_{k}(0)=\sqrt{2 \pi}\left(\frac{\Omega}{\Delta}\right) e^{-\left(\Omega^{2} / 2 \Delta^{2}\right)\left(2 \pi k / q_{0}\right)^{2}}
$$

only in the range $|k|<q_{0} \Delta /(2 \pi \Omega)$, i.e., for $\left|\zeta_{k}\right|<q_{0}(\Delta / \Omega) . F(\zeta, \tau)$ is normalized, $\int d \zeta F(\zeta, \tau)=$ $\sum N_{k}=1$. A somewhat more elaborate derivation of Eq. (40) is given in Appendix B.

The Fourier transform of the distribution function is the sum

$$
\tilde{F}(q, \tau)=\sum_{k} N_{k}(\tau) e^{-i q \zeta_{k}(\tau)}
$$

Such a sum is a sharp function of $\left|q-q_{0}\right|$. It can be written as

$$
\begin{aligned}
\tilde{F}(q, \tau) & =\tilde{F}\left(q_{0}, \tau\right) \Phi\left(q, q_{0}, \tau\right), \\
\Phi\left(q, q_{0}, \tau\right) & =\frac{\sum N_{k}(\tau) e^{-i q \zeta_{k}(\tau)}}{\sum N_{k}(\tau) e^{-i q_{0} \zeta_{k}(\tau)}} .
\end{aligned}
$$

Here

$$
\tilde{F}\left(q_{0}, \tau\right)=\int d \zeta d P F(\zeta, P, \tau) e^{-i q_{0} \zeta}
$$

and can be easily calculated numerically as will be shown below.

The factor $\Phi\left(q, q_{0}, \tau\right)$ can be determined using $N_{k}$, Eq. (41), and the estimate

$$
\sum_{k=-\infty}^{\infty} e^{-2 \pi i a k-b k^{2} / 2} \simeq \sqrt{\frac{2 \pi}{b}} e^{-\left(2 \pi^{2} / b\right)\{a\}^{2}}
$$

valid for small $0<b \ll 1$. The curly brackets here mean the fractional part of $a,\{a\}=a \bmod (1)$.

Calculations give

$$
\Phi\left(q, q_{0}, \tau\right) \propto e^{-(1 / 2)\left(q_{0} \Delta / \Omega\right)^{2}\left\{q\left[q_{0}\left(1+\Omega^{2}\right) / \omega^{2}\right]\right\}^{2}} .
$$

This is a narrow function around $q$ equal to multiples of $q_{0}$ with the width $\Delta q=\Omega / \Delta \ll 1$. The self-consistent potential is generated by the harmonic $q \simeq q_{0}$. Neglecting all other harmonics, Eq. (46) takes the form

$$
\Phi\left(q, q_{0}, \tau\right)=\Phi_{0}\left(q-q_{0}\right) e^{\left(q-q_{0} / q_{0}\right)\left[1-\left(\Omega^{2} / \omega^{2}\right)\right]\left[-i \nu \tau+\left(q_{0} \Delta / \omega\right)^{2}\right]},
$$


where

$$
\Phi_{0}\left(q-q_{0}\right)=e^{-(1 / 2)\left(q_{0} \Delta / \Omega\right)^{2}\left(q-q_{0}\right)^{2}} .
$$

Numeric calculations of $\Phi\left(q, q_{0}, \tau\right)$ show that the estimate Eq. (47) works very well for the parameters of interest. Now we can calculate $V(\zeta, \tau) \equiv w(\zeta, \tau)+$ c.c..

The Fourier harmonics $\tilde{w}(q, \tau)$ are defined by Eq. (7),

$$
\tilde{w}(q, \tau)=-\lambda^{3} \int_{-\infty}^{\tau} d \tau^{\prime} \tilde{F}\left(q, \tau^{\prime}\right) e^{i(q-1) \kappa\left(\tau-\tau^{\prime}\right)} .
$$

Substituting Eqs. (43) and (47), we get

$$
\tilde{w}(q, \tau)=-\frac{i}{2} \lambda^{3} \Phi_{0}\left(q-q_{0}\right) A\left(q, q_{0}, \tau\right)
$$

where

$$
A\left(q, q_{0}, \tau\right)=-2 i \int_{-\infty}^{\tau} d \tau^{\prime} \tilde{F}\left(q_{0}, \tau^{\prime}\right) e^{i\left(q_{0}-1\right) \kappa\left(\tau-\tau^{\prime}\right)} e^{\left(q-q_{0} / q_{0}\right)\left[1-\left(\Omega^{2} / \omega^{2}\right)\right]\left[-i \nu \tau^{\prime}+\left(q_{0} \Delta / \omega\right)^{2}\right]}
$$

Let us consider the short time interval, $\tau<\Delta /(\nu \Omega)$. Then, approximation $\Phi_{0}\left(q-q_{0}\right) \simeq\left(2 \pi / \lambda^{3}\right) \delta\left(q-q_{0}\right)$ gives

$$
\begin{gathered}
w(\zeta, \tau)=\int \frac{d q}{2 \pi} e^{i q \zeta} \tilde{w}(q, \tau)=-\frac{i}{2} A\left(q_{0}, q_{0}, \tau\right) e^{i q_{0} \zeta}, \\
A\left(q_{0}, q_{0}, \tau\right)=-2 i \int_{-\infty}^{\tau} d \tau^{\prime} \tilde{F}\left(q_{0}, \tau^{\prime}\right) e^{i\left(q_{0}-1\right) \kappa\left(\tau-\tau^{\prime}\right)}
\end{gathered}
$$

Comparison of the first equation of Eq. (52) with the assumed form $V(\zeta, \tau)=q_{0} a(\tau) \sin \left(q_{0} \zeta-\nu \tau\right)$ defines $a=A\left(q_{0}, q_{0}, \tau\right) e^{i \nu \tau}$.

The largest growth rate can be expected at $q_{0}=1$. To determine the slow dependence on time of real functions $a(\tau)$ and $\nu(\tau)$ in this case, we use the second equation of (52) in the differential form

$$
\begin{aligned}
\dot{a} & =i \nu a+2 i e^{i \nu \tau} \tilde{F}\left(q_{0}, \tau\right), \\
\tilde{F}\left(q_{0}, \tau\right) & =\sum_{k} N_{k} e^{-i q_{0} \zeta_{k}(\tau)} \simeq e^{-i\left[1-\left(\Omega^{2} / \omega^{2}\right)\right] \nu \tau} .
\end{aligned}
$$

Separating real and imaginary parts, we get two equations which can be solved for small $\tau \ll \tau_{\mathrm{dr}}$ where the time of the drift $\tau_{\mathrm{dr}} \simeq a /\left(\nu \Omega^{2}\right)$. In this case, the exponent in Eq. (53) can be expanded, giving

$$
a(\tau)=\left[6(\Omega \tau)^{2}\right]^{1 / 3}, \quad \nu=\frac{2}{a} .
$$

The amplitude growth depends on $\Omega$ and is much faster than that for the coasting beam [1]. The frequency $\nu$ decreases with time as $\nu \propto \tau^{-2 / 3}$.

\section{NUMERICAL SIMULATIONS}

In this section, we describe an approximate method based on Eqs. (52) and valid for $\tau<\tau_{\mathrm{dr}}$. A more general method is described in the next section.
In both cases, we replace the Fokker-Planck equation by the system of equations of motion for each of the $n_{\text {part }}$ test particles taking into account synchrotron motion:

$$
\frac{d \zeta}{d \tau}=P, \quad \frac{d P}{d \tau}=-\Omega^{2} \zeta-V(\zeta, \tau)-\Gamma_{s} P+\xi(\tau) .
$$

The synchrotron damping and fluctuations are taken into account in the second equation where $\xi$ is random variable,

$$
\langle\xi\rangle=0, \quad\left\langle\xi(\tau) \xi\left(\tau^{\prime}\right)\right\rangle=2 \Gamma \Delta^{2} \delta\left(\tau-\tau^{\prime}\right) .
$$

The interaction $V(\zeta, \tau)=2 \operatorname{Re}[w(\zeta, \tau)]$ for the time intervals $\tau<\tau_{\mathrm{dr}}$ can be approximated, see Eqs. (52), as

$$
\begin{aligned}
V(\zeta, \tau) & =\operatorname{Re}\left[-i A(\tau) e^{i q_{0} \zeta}\right], \\
\frac{d A}{d \tau} & =i\left(q_{0}-1\right) \kappa A-2 i \tilde{F}\left(q_{0}, \tau\right) .
\end{aligned}
$$

Here, $\tilde{F}\left(q_{0}, \tau\right)=\left\langle e^{-i q_{0} \tau}\right\rangle$ can be written in terms of the sum of the $n_{\text {part }}$ test particles,

$$
\left\langle e^{-i q_{0} \zeta}\right\rangle=\frac{1}{n_{\text {part }}} \sum_{k} e^{-i q_{0} \zeta_{k}(\tau)} .
$$

Note that we can expect that $A(\tau) \simeq a(\tau) e^{-i \nu \tau}$ where dependence of $a(\tau)$ was estimated in the previous section.

Equations (55), (57), and (58) are the full system of equations which were used for simulations.

Initial beam dynamics is the same as that described for the coasting beam [1]. Initial exponential growth predicted by the linear theory is followed at $\tau>1$ (i.e., $t>$ $\mu$ ) by oscillations with substantial growth of the rms energy spread.

Results of the long-range tracking of 1000 particles interacting with a single mode $q_{0}=1$ are shown in Figs. 4 

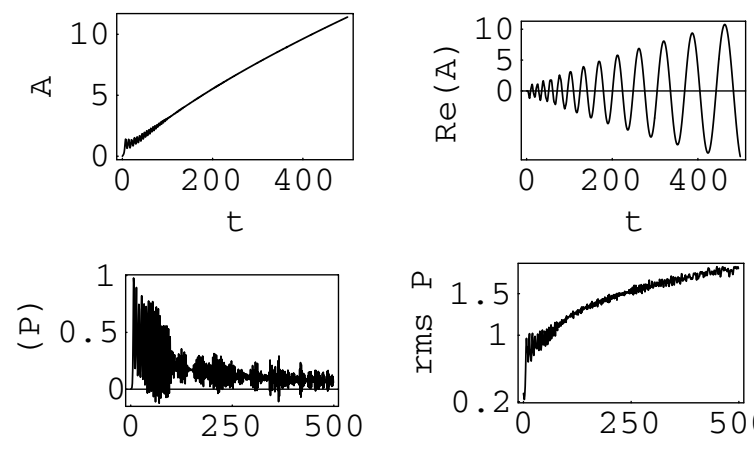

t
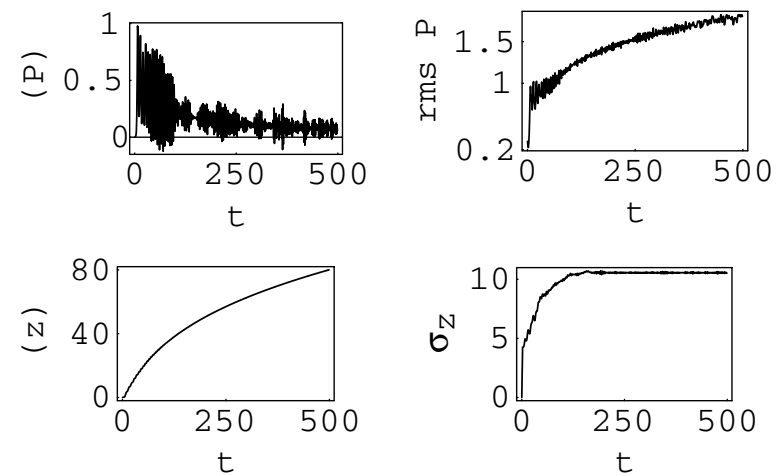

FIG. 4. Results of the tracking of 1000 particles with zero damping and detuning. Parameters: $A(0)=1.0 E-6, \Delta=0.3$, $\Omega=0.05$. Upper row: time dependence of $A(\tau)$ (left panel) and $\operatorname{Re} A(\tau)$ (right panel). Middle row: variation of the average momentum and the momentum rms with time. Bottom row: time dependence of the bunch centroid and the rms bunch length.

and 5. The following parameters were used in the simulation: $\Delta=0.3, \Omega=0.05$, and $\Gamma=0$. Initial amplitude $A(0)=1.0 \times 10^{-6}$ and particles were distributed in the phase plane with coordinates $\zeta(0)=\sqrt{2 J / \Omega} \sin \theta, P(0)=$ $\sqrt{2 J \Omega} \cos \theta$ uniformly within the area $-\pi<\theta<\pi$ and $0<J<\Delta^{2} / \Omega$.

In Fig. 4 the upper row shows a variation in time of the absolute value of the amplitude $|A(\tau)|$ and its real part. The absolute value of the amplitude varies as $|A(\tau)| \propto \tau^{\alpha}$ where $\alpha$ is between $2 / 3$ and $1 . \operatorname{Re}(A)$ oscillates with frequency $\nu$ which decreases while the amplitude grows. The second row shows the time dependence of the average and the rms momenta. The increase of the rms momentum follows the estimate Eq. (39). Parameter $\sigma_{z}$ depicted in Fig. 4 is the length of the train of microbunches rather than the rms width of the microbunch estimated in Eq. (39). The average bunch centroid and bunch length rms are shown in the bottom row. The distributions over $\zeta$ and $P$ are given in the bottom row. Figure 5 shows the phase plane and the distribution over $\zeta$ and momentum at the end of the tracking.

The results confirm the expected drift of particles to large amplitudes and increase of the rms momentum spread. The results have to be compared with the estimates Eq. (54) predicting the amplitude $a=15.5$, the tune $\nu=2 / a$, and the period of oscillations $2 \pi / \nu \simeq 90$ at the end of the run (at $\tau=500$ ). The growth of the rms momentum follows Eq. (54), and the distance of the drift $z_{\mathrm{dr}}=\nu \tau=64.5$ is in a good agreement with the estimate.
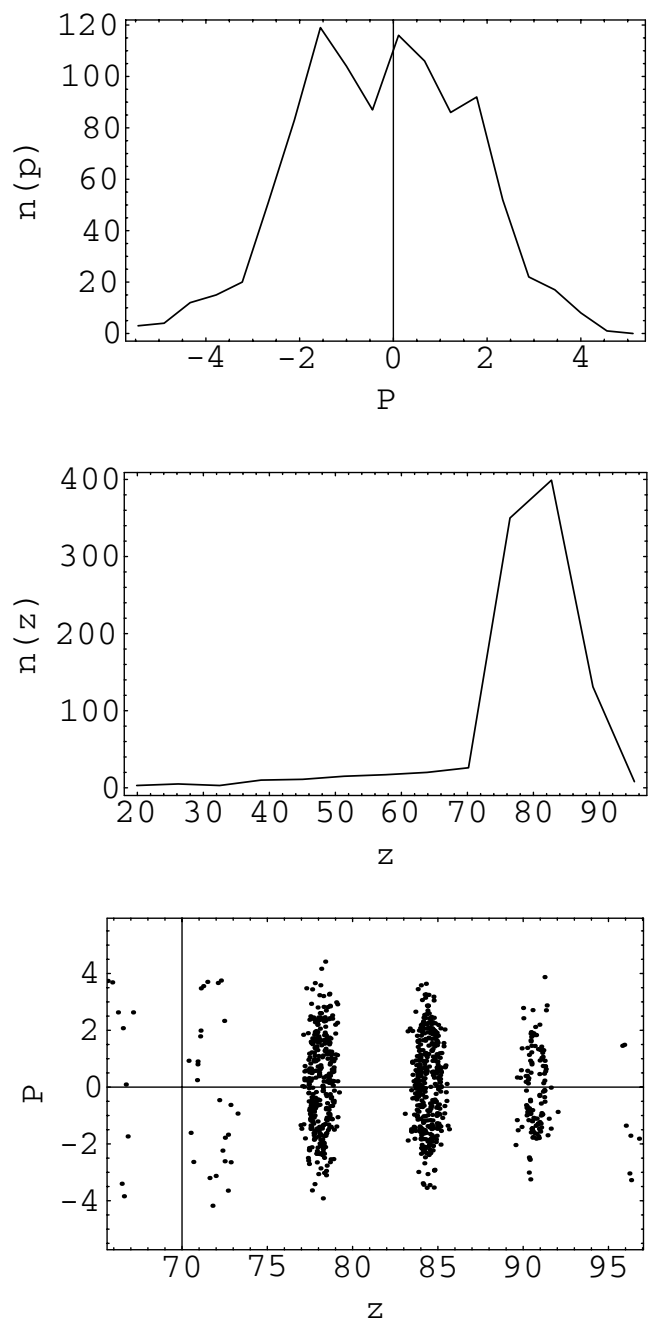

FIG. 5. Results of the tracking of 1000 particles with zero damping and detuning. Parameters are the same as in Fig. 4. From top to bottom: $n(P), n(\zeta)$, and the phase plane of a bunch, respectively, at the end of tracking, $\tau=500$.

Figure 6 compares the growth rate of the amplitude for several cases of $\Delta, \Omega$, and damping $\Gamma$. Results confirm that the growth rate depends mostly on $\Omega$ and is in reasonable agreement with the estimate Eq. (54). Figure 6(a) corresponds to $\Delta=0.03, \Gamma=0$ and two values of $\Omega=0.05$ (upper curve) and $\Omega=0.01$ (lower curve). Equation (54) predicts in the first/second case $|A|=15.5$ and $|A|=5.3$ at $\tau=500$. In both cases $6(\mathrm{a})$ and 6(b) $\Omega=0.005$ is the same, but in case 6(b) $\Delta=0.3$ is 10 times larger than in 6(c) without an effect on the growth rate. Effect of the damping is very small and illustrated by 6(d) where damping is turned on, $\Gamma=$ 0.01 while $\Delta=0.03$, and $\Omega=5 E-4$. Such a small $\Omega$ does not give any noticeable growth.

The results of numeric simulations discussed above are in good agreement with the analytic estimates of the previous section. One should remember, however, that the simulations are based on the equations which are 

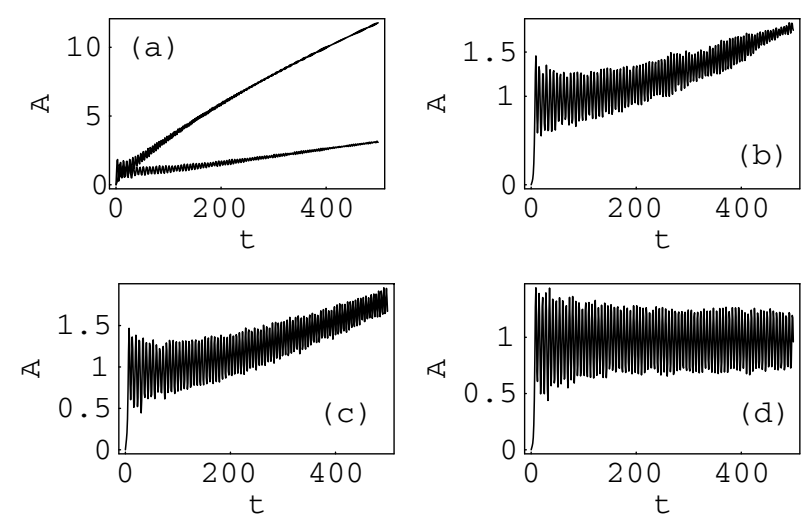

FIG. 6. Dependence of the growth rate on parameters. (a) $\Delta=0.03, \Gamma=0$, and $\Omega=0.05$ (upper curve) and $\Omega=0.01$ (lower curve). Equation (54) predicts at $\tau=500,|A|=15.5$, and $|A|=5.3$, respectively. (b) $\Omega=0.005, \Delta=0.3$, and $\Gamma=$ 0 . (c) $\Omega=0.005, \Delta=0.03$, and $\Gamma=0$. (d) $\Delta=0.03, \Omega=$ $5 E-4$, and the damping is on, $\Gamma=0.01$.

valid only at $\tau<\tau_{\mathrm{dr}} \simeq a /\left[\Omega^{2} \nu(0)\right]$, where $\nu(0)$ is the coherent tune shift of the linear approximation.

In the next section we study whether there is an asymptotic steady-state regime.

\section{FULL SIMULATIONS}

For large time intervals $\tau>\tau_{\mathrm{dr}}$, most of the initially trapped particles are released and start synchrotron oscillations with large amplitudes. The approximation Eq. (57) cannot be used in this case and simulations have to be based on the exact expression Eq. (7) for $V(\zeta, \tau)$. Changing variables, we write $V$ as

$$
V(\zeta, \tau)=-\frac{\lambda^{3}}{\kappa} \int_{\zeta}^{\infty} d \zeta^{\prime} F\left(\zeta^{\prime}, \tau-\frac{\zeta^{\prime}-\zeta}{\kappa}\right) e^{-i\left(\zeta-\zeta^{\prime}\right)}+\text { c.c. }
$$

In the simulations, we use a mesh covering the range $-20 \sigma_{\zeta}<\zeta<20 \sigma_{\zeta}$, where $\sigma_{\zeta}=\Delta / \Omega$ is the initial rms bunch length. The step size is chosen to have 12 mesh lines per interval $\Delta \zeta=2 \pi$. That gives a good enough resolution within the expected wavelength of perturbation $\lambda=2 \pi / q$ with $q=1$. The total number of mesh points was rounded to $n_{m}=512$ to be suitable for Fourier analysis.

Initial distribution of particles in the phase space was the same as in the previous section. To avoid the artificial excitation of bunch centroid motion, the set $\zeta_{k}, P_{k}$ was corrected to put the average $\langle\zeta\rangle$ and $\langle P\rangle$ to zero.

The time evolution was obtained by solving the equation of motion (55) with the time steps $\Delta \tau$ for each of the $M$ test particles. The solution on each step was obtained using the subroutine from the FORTRAN IMSL library and the synchrotron radiation diffusion and damping were taken into account at the end of a step by changing variables $p$ of each particle:

$$
p_{k} \rightarrow p_{k}-\Gamma \Delta \tau p_{k}+\sqrt{24 \Delta \tau \Gamma \overline{\Delta^{2}}} \xi
$$

Here $\xi$ is a random variable uniformly distributed in the interval $-1 / 2<\xi<1 / 2$ and $k=1,2, \ldots, M$. At each time step, the particle density $F(\zeta)$ was calculated at the mesh points allocating each test particle to the two closest mesh points. An attempt was also made to refine the result changing the width of the bins according to the initial bin population, recalculating the population of the new bins, and recalculating the distribution function.

The integral in Eq. (59) was calculated using 3-meshpoint interpolation method. The time step $\Delta \tau=\Delta \zeta / \kappa$ was used to simplify the bookkeeping: with such a choice, the shift by one mesh step $\Delta \zeta$ corresponds also to the shift by one time step $\Delta \tau$. The density $F(\zeta, \tau)$ was stored as a matrix $n_{m} \times n_{m}$ and updated at each time step.

Results of simulations depicted below are obtained for the following parameters: $\Delta=0.3, \Omega=0.05, \kappa=10.0$, and $\Gamma=0.01$. The number of the test particles in simulations was $M=10^{5}$. The total number of time steps, 15000 , corresponds to the time interval of more than seven damping times. The CPU time for simulations with these parameters is $1.3 \mathrm{~h}$ on $476 \mathrm{MHz} \mathrm{PC}$.

The time evolution of the average momentum and position of the bunch centroid as well as time dependence of the rms of momentum spread and bunch length are shown in Fig. 7.

Distribution of particles in the phase plane $(\zeta, P)$ is shown in Fig. 8 at $\tau=0$ and at $\tau=31.5$, when the instability already substantially distorted the distribution. The snapshots of the phase plane at the end of simulations, $\tau \simeq 700$, are shown in Fig. 9 with the time interval of a quarter of synchrotron period.

The snapshots of the bunch density $F(\zeta, \tau)$ are depicted in Fig. 10 with the time intervals of a quarter of synchrotron period $T_{s} / 4=2 \pi /(4 \Omega)$. The periodic modulation of the density profile is clearly seen in the initial steps of the simulations. The amplitude of the modulation decreases
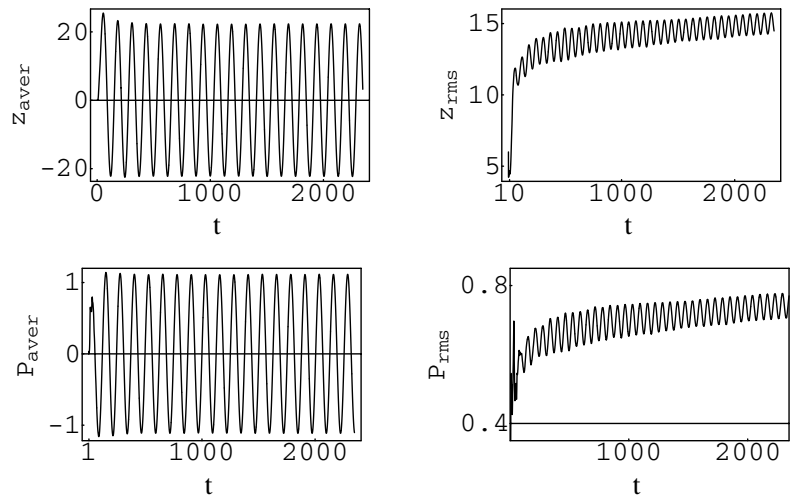

FIG. 7. The variation of the average momentum, position of the bunch centroid, the rms energy spread, and the rms bunch length with time. The fast initial growth is replaced by the synchrotron oscillations with large amplitudes. 

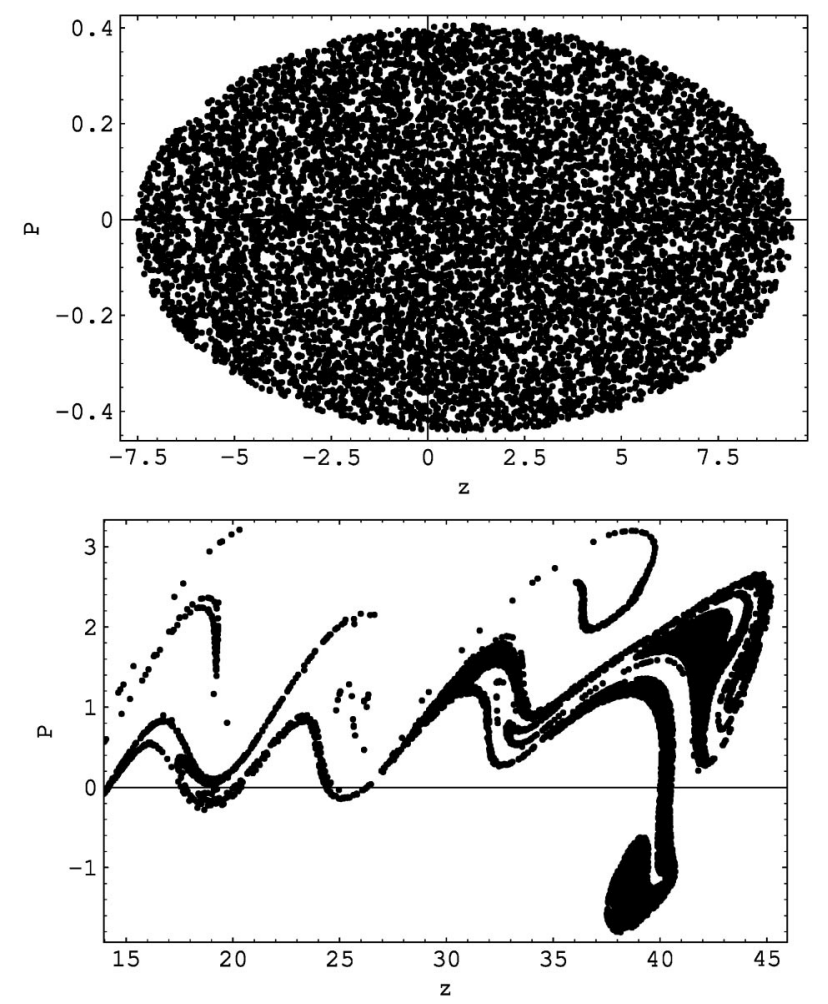

FIG. 8. The distribution of particles in the phase plane: initial, $\tau=0$, (top panel) and at $\tau=31.5$.

with time but does not disappear completely due to the distortion at the periphery of the distribution in the phase plane; see Fig. 9. Note also the change of the range in the $\zeta$ axes indicating the synchrotron motion of the bunch centroid.

The first several snapshots of $V(\zeta, \tau)$ vs $\zeta$ (left column) and the Fourier spectrum $\tilde{V}(q, \tau)$ vs $q$ (right column) taken with the time interval $\pi /(4 \Omega)$ are shown in Fig. 11. The spectrum is centered at $q \simeq 1$, but become wider with time. The maximum amplitude of $V(\zeta, \tau)$ decreases with time. The time dependence of $V(\tau)$ has some similarity with simulations for continuous spectrum [4] but several peaks in the beginning are transient and disappear later. It is worth noting that these peaks are not artifact or noise: in the interval $0<\tau<100$ in Fig. 12 there are 2000 data points.

\section{CONCLUSION}

We studied the beam dynamics affected by the microwave CSR generated by relatively long bunches in storage rings close to the shielding threshold where it can be dominated by a single EM mode excited in the beam pipe. In the dimensionless variables, there are only a few parameters $\Delta, \Omega$, and $\kappa$, which determine the instability. For high frequency perturbations, the ratio $\Omega / \Delta \ll 1$. The linear theory predicts strong instability and generation of the EM wave with the exponentially growing amplitude where the growth rate is larger than
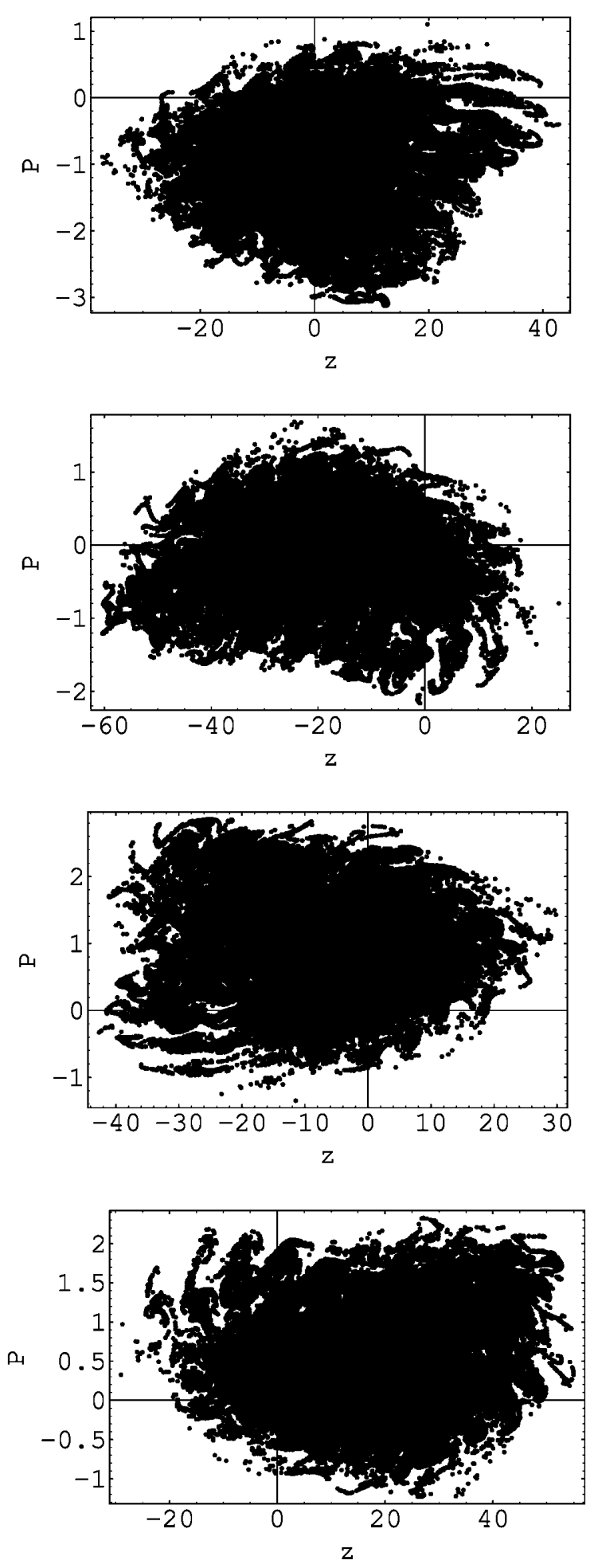

FIG. 9. The snapshots of the phase plane at $\tau \simeq 700$ with the time interval $T_{s} / 4$. Note the difference in the position of the bunch centroid.

the synchrotron frequency. We tried to understand whether such growth may lead to a steady-state saturation regime that would have interesting experimental implications. We show that, in the nonlinear regime, the 

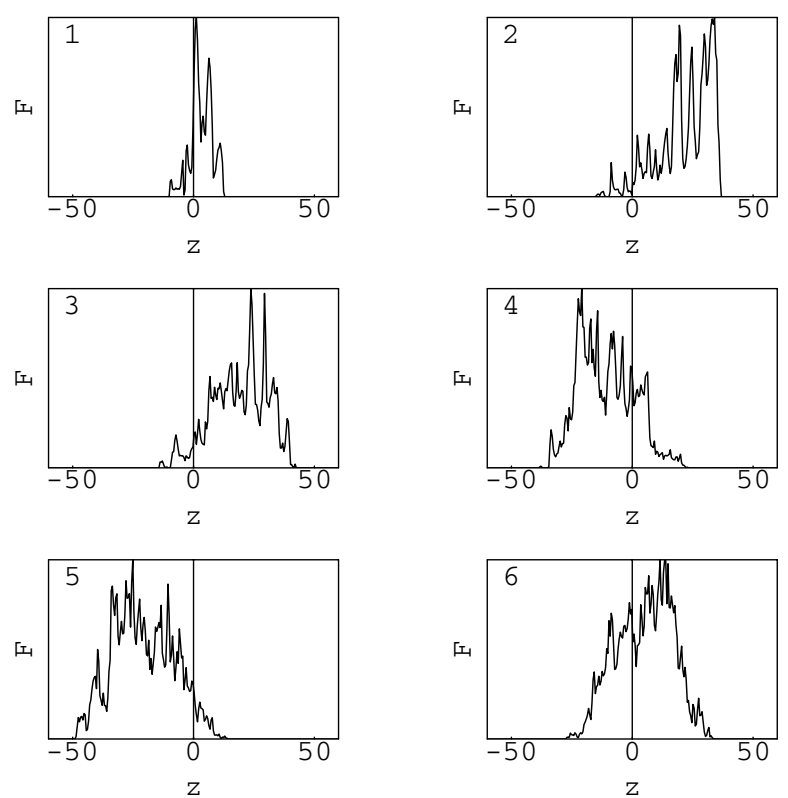

FIG. 10. The bunch density $F(\zeta, \tau)$ depicted with the time interval $T_{s} / 8$. Note the difference in the position of the bunch centroid.

synchrotron motion substantially affects the instability. After initial exponential growth of the linear regime at the time intervals $\tau<\simeq 1$, the beam dynamics is substantially different in the cases of the time intervals smaller and larger than the drift time $\tau_{\mathrm{dr}}$. In the first interval, particles are trapped by a fast growing wave leading to the formation of a chain of microbunches. The particle density is strongly modulated with the wavelength of the
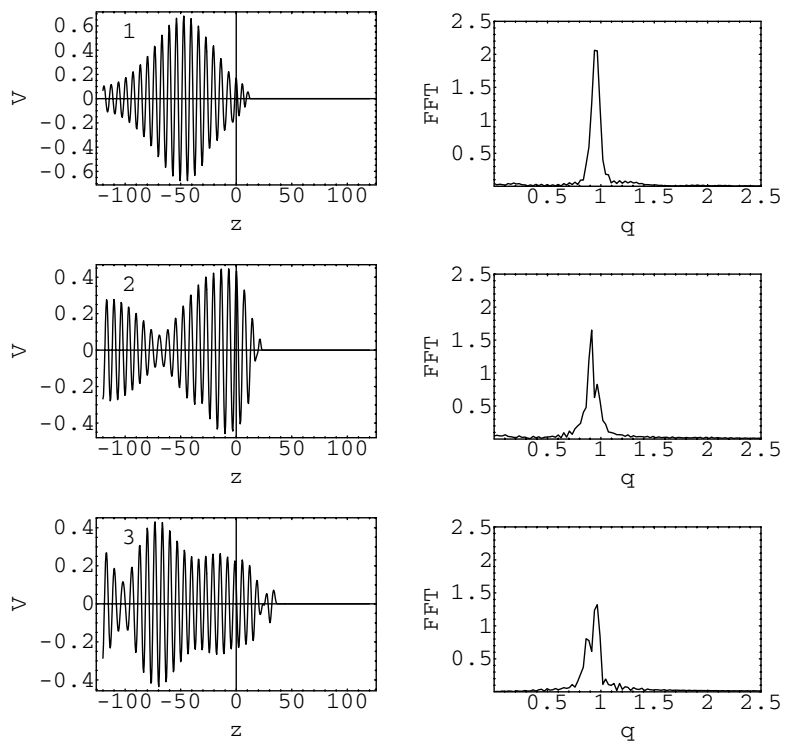

FIG. 11. The first several snapshots of $V(\zeta, \tau)$ vs $\zeta$ (left column) and the Fourier spectrum $\tilde{V}(q, \tau)$ vs $q$ (right column) taken with the time interval $\pi /(4 \Omega)$. The spectrum is centered at $q \simeq 1$, but become wider with time.
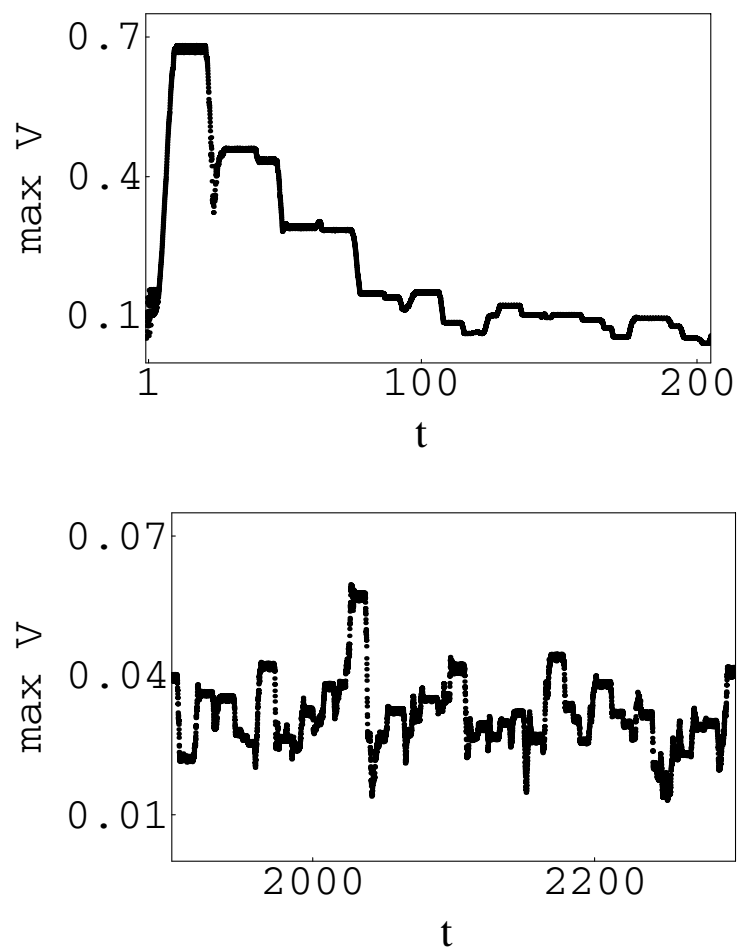

FIG. 12. The maximum amplitude of $V(\zeta, \tau)$ vs $\tau$ displayed for two time intervals. Data taken at each time step: about 2000 data points in the interval $0<\tau<100$. Initially, there are several picks which become smaller later but do not disappear completely.

mode. The energy spread of particles is much larger than the initial rms $\Delta$. The interaction of a chain of trapped particles with the wave is strong and the wave amplitude $V$ grows in time faster than in the case of a coasting beam. Later, for $\tau>\tau_{\mathrm{dr}}$, most of the particles are pulled to the edge of the rf potential, released, and start synchrotron oscillations with large amplitudes. Because trapped particle are released asymmetrically only at one edge of the rf potential well, strong oscillations of the bunch centroid are excited. The released particles do not form a regular pattern in the bunch density. The modulation of the bunch distribution is visible only at the periphery of the phase plane and the amplitude of the generated wave $V$ decreases. The remnant amplitude, nevertheless, is sufficiently large to compensate the synchrotron radiation damping and support the large energy spread in the bunch. There are several peaks in $V(\tau)$ dependence but they are transient phenomena.

If particles survive, they can be trapped again due to the radiation damping. However, the trapping and the drift of trapped particles both lead to a large rms energy spread and to oscillations of particles with large synchrotron amplitudes. In the beam pipe with a finite aperture that would lead to particle loss. Probably, a betatron with the coasting beam may have an advantage compared to the bunched beam in synchrotrons for a machine designed as a source of microwave radiation. 


\section{ACKNOWLEDGMENTS}

I thank G. Stupakov and K. Bane for their help and useful discussions. This work was supported by the Department of Energy Contract No. DE-AC0376 SF00515.

\section{APPENDIX A: INTERACTION OF A BEAM WITH A SINGLE MODE}

The equation for electric field can be obtained analogously to one given for the coasting beam, see Appendix A,

$$
\mathcal{E}(z, t)=\frac{i e\left(1-\beta_{g}\right) \chi}{\beta_{g}} \int d z^{\prime} d t^{\prime} f\left(z^{\prime}, t^{\prime}\right) \int \frac{d q}{(2 \pi)} e^{i q\left[z-z^{\prime}+c\left(t-t^{\prime}\right)\right]} \int \frac{d \omega}{(2 \pi)} \frac{e^{-i \omega\left(t-t^{\prime}\right)}}{q-q(n, \omega)-i \epsilon}
$$

Integration over $\omega$ is defined by the contribution of the pole $\omega_{n}(q)=q$,

$$
\int \frac{d \omega}{2 \pi} \frac{e^{-i \omega\left(t-t^{\prime}\right)}}{q-q(n, \omega)-i \epsilon}=i v_{n}(q) \Theta\left(t-t^{\prime}\right) e^{-i \omega_{n}(q)\left(t-t^{\prime}\right)}
$$

where $v_{n}(q)=d \omega_{n}(q) / d q$, and $\Theta\left(t-t^{\prime}\right)$ is the step function.

The force $\mathcal{E}(z, t)=e E(c t+z, t)$ acting on the trailing particle with displacement $z$ from the bunch centroid $(z>0$ is in the head of the bunch) is given by the longitudinal component $E(s, t)$ excited by the bunch,

$$
\mathcal{E}(z, t)=-e^{2} c\left(1-\beta_{g}\right) N_{b} \chi_{n} \int d t^{\prime} d z^{\prime} f\left(z^{\prime}, t^{\prime}\right) \Theta\left[t-t^{\prime}\right] \int \frac{d q}{2 \pi} e^{i q(c t+z)-i \omega_{n}(q) t} e^{-i q\left(c t^{\prime}+z^{\prime}\right)+i \omega_{n}(q) t^{\prime}}+\text { c.c. }
$$

Here $f(z, t)$ is the distribution function of the bunch normalized by the condition $\int d z f(z, t)=1$, and the coefficient is determined using the definition of the loss factor $\chi_{n}$ of the $n$th mode for a pointlike bunch, $f(z, t)=\delta(z)$. Equation (A3) shows that the sign of $\epsilon>0$ in Eq. (A2) corresponds to the condition of casuality.

Neglecting dispersion $d^{2}(\omega / c) / d q^{2} \simeq b^{3} / R^{2}$ (where $b$ is the beam pipe radius and $R$ is the bend radius), we can expand $\omega_{n}(q)=q_{n} c+\left(q-q_{n}\right) v_{g}$. Here $v_{g}$ and $\omega_{n}=q_{n} c$ are the group velocity and frequency of the synchronous component of the $n$th mode, respectively. Then

$$
\mathcal{E}(z, t)=-e^{2} c\left(1-\beta_{g}\right) N_{b} \chi_{n} \int_{-\infty}^{t} d t^{\prime} f\left[z+c\left(1-\beta_{g}\right)\left(t-t^{\prime}\right), t^{\prime}\right] e^{-i q_{n} c\left(1-\beta_{g}\right)\left(t-t^{\prime}\right)}+\text { c.c. }
$$

In the steady state, where $f(z, t)=f(z)$,

$$
\mathcal{E}=-e^{2} N_{b} \chi \int_{z}^{\infty} d z^{\prime} f\left(z^{\prime}\right) e^{-i q_{n}\left(z^{\prime}-z\right)}+\text { c.c. }
$$

In the dimensionless variables, see Eqs. (3) and (5), the interaction $V$ in Eq. (9) in this case is

$$
V=-\frac{d P}{d \tau}=-\frac{\lambda^{3}}{\kappa} \int_{\zeta}^{\infty} d \zeta^{\prime} f\left(\zeta^{\prime}\right) e^{i\left(\zeta-\zeta^{\prime}\right)}+\text { c.c. }
$$

\section{APPENDIX B: INITIAL FORM OF THE DISTRIBUTION FUNCTION}

We want to show that $F(\zeta, \tau)$ is consistent with the form of the potential $U$, Eq. (34) during the time less than the drift time $\tau_{\mathrm{dr}}$, provided $a$ and $\nu$ are slowly varying with time.

Neglecting slow damping and diffusion, we can replace the Fokker-Planck equation by the system of equation of motion (33) (with $\Gamma=0$ ). It is natural to describe the motion of a particle trapped in the $k$ th microwell by the coordinates $x, \dot{x}$,

$$
x=q_{0} \zeta-\nu \tau-2 \pi k, \quad \dot{x}=Q=q_{0} P-\nu .
$$

The oscillations of a particle are defined by the equation

$$
\frac{d^{2} x_{k}}{d \tau^{2}}+\Omega^{2} x+a q_{0}^{2} \sin x=-\Omega^{2}(2 \pi k+\nu \tau) .
$$

For large $a$, the amplitude of oscillations is small, and $\sin x \simeq x$. Then, Eq. (B2) describes a linear oscillator with the frequency $\omega, \omega^{2}=q_{0}^{2} a+\Omega^{2}$ and has the solution 


$$
\begin{aligned}
& x\left(x_{0}, Q_{0}, \tau\right)=-\left(\frac{\Omega}{\omega}\right)^{2}(2 \pi k+\nu \tau)+\left[x_{0}+2 \pi k\left(\frac{\Omega}{\omega}\right)^{2}\right] \cos (\omega \tau)+\frac{1}{\omega}\left[Q_{0}+\nu\left(\frac{\Omega}{\omega}\right)^{2}\right] \sin (\omega \tau), \\
& Q\left(x_{0}, Q_{0}, \tau\right)=-\nu\left(\frac{\Omega}{\omega}\right)^{2}-\omega\left[x_{0}+2 \pi k\left(\frac{\Omega}{\omega}\right)^{2}\right] \sin (\omega \tau)+\left[Q_{0}+\nu\left(\frac{\Omega^{2}}{\omega}\right)^{2}\right] \cos (\omega \tau) .
\end{aligned}
$$

Here, $x_{0}$ and $Q_{0}$ are constant integrals of motion. They are related to the initial conditions $\zeta(0)=\zeta_{0}$ and $P(0)=P_{0}$ at $\tau=0$,

$$
x_{0}=q_{0} \zeta_{0}-2 \pi k, \quad Q_{0}=q_{0} P_{0}-\nu .
$$

The initial Gaussian bunch $F(\zeta, P, 0) \propto \exp \left[-\left(1 / \Delta^{2}\right)\left(P_{0}^{2}+\Omega^{2} \zeta_{0}^{2}\right)\right]$. At $\tau>0$,

$$
F(\zeta, P, \tau)=\sum_{k} \int d \zeta_{0} d P_{0} F\left(\zeta_{0}, P_{0}, 0\right) \delta\left[\zeta-\frac{1}{q_{0}}\left(x\left(\zeta_{0}, P_{0}, \tau\right)+\nu \tau+2 \pi k\right)\right] \delta\left[P-\frac{1}{q_{0}}\left(Q\left(\zeta_{0}, P_{0}, \tau\right)+\nu\right)\right],
$$

where $x\left(\zeta_{0}, P_{0}, \tau\right)$ and $Q\left(\zeta_{0}, P_{0}, \tau\right)$ after substitution of $x_{0}$ and $Q_{0}$ from Eq. (B4) are defined by Eq. (B3).

Integrating Eq. (B5) and averaging terms oscillating with the frequency $\omega$, we get $F(\zeta, P, \tau)$. After the additional integration over $P$,

$$
F(\zeta, \tau)=\frac{\Omega}{q_{0} \Delta^{2}} \sqrt{\frac{\omega^{2}+\Omega^{2}}{2}} \sum_{k} e^{-\left(\omega^{2}+\Omega^{2} / 4 \Delta^{2}\right)\left[\zeta-(\nu \tau+2 \pi k) / q_{0}\left(1+\Omega^{2} / \omega^{2}\right)\right]^{2}-(1 / 2)\left(2 \pi k \Omega / q_{0} \Delta\right)^{2}},
$$

where small terms of the order of $(\nu / \omega)^{2}(\Omega / \Delta)^{2} \ll 1$ and $(2 \pi k)^{2} \Omega^{4} /(\Delta \omega)^{2}<(\Omega / \omega)^{2} \ll 1$ are neglected.

Equation (B6) is valid for $\left.(\Omega / \omega)^{2} \mid \nu \tau+2 \pi k\right) \mid<1$; that is, for $\tau<\tau_{\mathrm{dr}}=\omega^{2} /(\nu \Omega)$ provided $\omega \gg \Omega$.

For large $a \gg \Delta^{2}$, the normalized distribution function Eq. (B6) can be written as a sum of $\delta$ functions,

$F(\zeta, \tau)=\sum_{k} \sqrt{2 \pi} \frac{\Omega}{q_{0} \Delta} e^{-2\left(\pi k \Omega / q_{0} \Delta\right)^{2}} \delta\left[\zeta-\frac{\nu \tau+2 \pi k}{q_{0}\left(1+\Omega^{2} / \omega^{2}\right)}\right]$.

This form was assumed in Eq. (40).
[1] S. Heifets and G. Stupakov, SLAC Report No. SLACPUB-9627, 2003.

[2] S. Heifets and G. Stupakov, Phys. Rev. ST Accel. Beams 5, 054402 (2002).

[3] Proceedings of the Workshop on Coherent Synchrotron Radiation in Storage Rings, Napa, CA, 2002 (unpublished).

[4] M. Venturini and R. Warnock, Phys. Rev. Lett. 89, 224802 (2002). 